\title{
The Deep X-ray Radio Blazar Survey (DXRBS)
}

\author{
Paolo Padovani ${ }^{1,2,3}$, Eric Perlman ${ }^{1}$, Paolo Giommi ${ }^{4}$, Rita Sambruna ${ }^{5}$, Laurence \\ R. Jones ${ }^{6}$, Anastasios Tzioumis ${ }^{7}$ and John Reynolds ${ }^{7}$ \\ 1 Space Telescope Science Institute, 3700 San Martin Drive, Baltimore MD. 21218, \\ USA \\ 2 Affiliated to the Astrophysics Division, Space Science Department, European Space \\ Agency \\ 3 On leave from Dipartimento di Fisica, II Università di Roma "Tor Vergata", Italy \\ 4 SAX Science Data Center, ASI, Viale Regina Margherita 202, I-00198, Italy \\ 5 Pennsylvania State University, Department of Astronomy, 525 Davey Lab, University \\ Park, PA 16803 \\ 6 School of Physics \& Astronomy, Univ. of Birmingham, Birmingham B15 2TT, UK \\ 7 Australia Telescope National Facility, CSIRO, PO Box 76, Epping NSW 2121, Aus- \\ tralia
}

\begin{abstract}
We have undertaken a survey for blazars by correlating the ROSAT WGACAT database with publicly available radio catalogs, restricting our candidate list to serendipitous flat-spectrum sources $\left(\alpha_{\mathrm{r}} \leq 0.7, f_{\nu} \propto \nu^{-\alpha}\right)$. We discuss here our survey methods, identification procedure and first results. Our survey is found to be $\sim 95 \%$ efficient at finding blazars, a figure which is comparable to or greater than that achieved by other radio and X-ray survey techniques. DXRBS provides a much more uniform coverage of the parameter space occupied by blazars than any previous survey. Particularly important is the identification of a large population of flat-spectrum radio quasars with ratios of X-ray to radio luminosity $\gtrsim 10^{-6}\left(\alpha_{\mathrm{rx}} \lesssim 0.78\right)$ and of many low-luminosity flat-spectrum radio quasars. Moreover, DXRBS fills in the region of parameter space between X-ray selected and radio-selected samples of BL Lacs.
\end{abstract}

\section{Introduction}

Blazars are the most extreme variety of Active Galactic Nuclei (AGN) known. Their signal properties include irregular, rapid variability; high optical polarization; core-dominant radio morphology; apparent superluminal motion; flat $\left(\alpha_{\mathrm{r}} \lesssim 0.5\right)$ radio spectra; and a broad continuum extending from the radio through the gamma-rays (e.g., Urry \& Padovani 1995). The broadband emission from blazars is dominated by non-thermal processes (most likely synchrotron and inverse-Compton radiation). Blazar properties are consistent with relativistic beaming, that is bulk relativistic motion of the emitting plasma at small angles to the line of sight (as originally proposed by Blandford \& Rees in 1978), which gives rise to strong amplification and collimation in the observer's frame. It then follows that an object's appearance depends strongly on orientation. Hence the need for "Unified Schemes", which look at intrinsic, isotropic properties, to unify fundamentally identical (but apparently different) classes of AGN. 
The blazar class includes flat-spectrum radio quasars (FSRQ) and BL Lacertae objects. The main difference between the two classes lies in their emission lines, which are strong and quasar-like for FSRQ and weak or in some cases outright absent in BL Lacs. As a consequence of their peculiar orientation with respect to our line of sight, blazars represent a very rare class of objects, making up considerably less than 5\% of all AGN (Padovani 1997).

\section{Why a Blazar Survey?}

A blazar survey is needed essentially for two reasons: number statistics and limiting fluxes. All existing blazar samples, in fact, are relatively small and at relatively high fluxes. The largest radio-selected BL Lac sample, the 1 Jy sample (Stickel et al. 1991), includes 37 objects with $f_{5 \mathrm{GHz}}>1 \mathrm{Jy}$. In the X-rays, there are a few available samples, made up of 40 - 70 objects (the EMSS sample [Maccacaro et al. 1994], the Slew sample [Perlman et al. 1996], plus various ROSAT-based samples [Laurent-Muehleisen et al. 1997; Bade et al., in preparation]) but the deepest sample is still the EMSS one, which reaches $f_{\mathrm{x}} \sim 2 \times 10^{-13}$ $\mathrm{erg} \mathrm{cm}^{-2} \mathrm{~s}^{-1}$. (See however Wolter et al. 1997 for an ongoing deeper survey.)

Moving to FSRQ, until very recently the only sizeable sample with complete redshift information was the one extracted from the 2 Jy sample (Wall \& Peacock 1985), which includes 52 sources. (The FSRQ in the 1 Jy and S4 samples reach lower fluxes [ $1 \mathrm{Jy}$ and $0.5 \mathrm{Jy}$ respectively] and are more numerous but the identification of the two samples is still not complete.) Drinkwater et al. (1997) have recently published the PKS 0.5 Jy sample, which includes 323 flatspectrum radio sources with $f_{2.7 \mathrm{GHz}}>0.5 \mathrm{Jy}, 86 \%$ of which have a measured redshift. Finally, Shaver, Hook, and collaborators have just put together a sample of more than 400 flat-spectrum objects down to $f_{2.7 \mathrm{GHz}}=0.25 \mathrm{Jy}$ (Hook, these proceedings).

There is then clearly a need for a deeper, larger blazar survey, to address many open questions of blazar research, namely:

- BL Lac evolution and luminosity functions; unified schemes

- Flat-spectrum radio quasar evolution and luminosity functions; unified schemes

- The relationship between BL Lacs selected in the radio and X-ray band

- The relationship between flat-spectrum radio quasars and BL Lacs

- What is a BL Lac?

- The relationship between physical parameters (e.g., X-ray and radio spectral indices, equivalent width, line luminosity, continuum luminosity, etc.)

\section{The DXRBS}

The basic idea behind our Deep X-ray Radio Blazar Survey (DXRBS) is quite simple: blazars are relatively strong X-ray and radio emitters so selecting Xray and radio sources with flat radio spectrum (one of their defining properties) 
should be a very efficient way to find these rare sources. We adopt a spectral index cut $\alpha_{\mathrm{r}} \leq 0.7$. This will: 1 . select (by definition!) flat-spectrum radio quasars; 2 . select basically $100 \%$ of BL Lacs; 3. exclude the large majority of radio galaxies.

We have then cross-correlated WGACAT (White, Giommi \& Angelini 1995), the publicly available database of ROSAT PSPC sources (restricting ourselves to sources having quality flag $\geq 5$ to avoid problematic detections) with a number of publicly available radio catalogs. North of the celestial equator, we used the $20 \mathrm{~cm}$ and $6 \mathrm{~cm}$ Green Bank survey catalogs NORTH20CM and GB6 (White \& Becker 1992; Gregory et al. 1996), while south of the equator, we used the Parkes-MIT-NRAO catalog PMN (Griffith \& Wright 1993). All sources with radio spectral index $\alpha_{\mathrm{r}} \leq 0.7$ at a few $\mathrm{GHz}$ were selected as blazar candidates. Note that WGACAT reaches $f_{\mathrm{x}} \sim 10^{-14} \mathrm{erg} \mathrm{cm}^{-2} \mathrm{~s}^{-1}$ (although its flux limit varies widely on the sky), while the flux limits of the radio catalogs are the following: $f_{5 \mathrm{GHz}} \sim 20 \mathrm{mJy}$ (GB6), $f_{1.4 \mathrm{GHz}} \sim 100 \mathrm{mJy}$ (NORTH20CM), and $f_{5 \mathrm{GHz}} \sim 40 \mathrm{mJy}(\mathrm{PMN})$. (See Fig. 1 and section 4.)

For objects north of the celestial equator, $6-20 \mathrm{~cm}$ radio spectral indices were obtained directly from the cross-correlation of the GB6 and NORTH20CM catalogs. For sources at southern declinations, the lack of a comparably deep radio survey at a second frequency required a different strategy ${ }^{1}$. We then conducted a snapshot survey with the Australia Telescope Compact Array (ATCA) at 3.6 and $6 \mathrm{~cm}$, to get also radio spectral indices unaffected by variability (which will be a problem for our northern sample).

As a result of the correlations between the X-ray and the radio catalogs we have obtained a list of about 200 blazar candidates (with $|b|>10^{\circ}$ ), to which we add 88 previously known, serendipitous (i.e., not ROSAT targets) blazars (77 FSRQ and 11 BL Lacs), for a total of about 300 sources.

We note that, as the original catalogs included tens of thousands of objects, our search strategy has narrowed down the number of candidates by more than two orders of magnitude. This kind of approach is extremely important for surveys, like ours, that look for rare objects in large catalogs and will be vital with the advent of even larger and deeper catalogs, foreseen in the near future.

\subsection{The Identifications}

Accurate positions to pinpoint the optical counterparts were obtained from either the NVSS (Condon et al. 1997) or our ATCA survey. Magnitudes for all X-ray/radio sources with counterparts on the POSS and UKST plates which comprise the Digitized Sky Survey were obtained from the Cambridge APM and Edinburgh COSMOS projects (Irwin et al. 1994; Drinkwater et al. 1995). Most $\mathrm{X}$-ray/radio sources without counterparts on the survey plates were imaged at either the KPNO $0.9 \mathrm{~m}$ or the CTIO $0.9 \mathrm{~m}$ telescopes. This allowed identification of optical counterparts to $R=23$. The magnitude distribution of the blazar

\footnotetext{
1 The NVSS survey (Condon et al. 1997) was not available when we started this project. Moreover, it is still not $100 \%$ completed and covers the sky north of $-40^{\circ}$.
} 
candidates peaks around 18. Spectroscopic observations were conducted at the KPNO $2.1 \mathrm{~m}$, MMT, Lick $3 \mathrm{~m}$, ESO $2.2 \mathrm{~m}$ and $3.6 \mathrm{~m}$, and CTIO $1.5 \mathrm{~m}$ telescopes.

Table 1. DXRBS Identifications

\begin{tabular}{lrrr}
\hline Class & Newly identified & Previously known & Total \\
\hline Radio Quasars & 86 & 77 & 163 \\
BL Lacs & 26 & 11 & 37 \\
Radio Galaxies & 4 & 10 & 14 \\
\hline Total & 116 & 98 & 214 \\
\hline
\end{tabular}

The breakdown of the identifications at the time of writing (February 1998) is given in Table 1. So far we have identified $\sim 50 \%$ of our candidates and $97 \%$ of them are blazars. $90 \%$ of the previously known objects are also blazars ${ }^{2}$. Our method is then indeed very efficient (93.5\%) at identifying blazars.

\section{First Results}

The 163 DXRBS FSRQ we have so far indentified span the redshift range of 0.1 to 3.8 , with a mean value of 1.25 . Figure 1 a shows the greatly improved coverage of the radio/X-ray flux plane for FSRQ provided by DXRBS, as compared to previously available samples (note that both the 1 Jy and S4 samples are not completely identified and X-ray data are available only for $\sim 60 \%$ of their FSRQ). DXRBS FSRQ go about an order of magnitude deeper in radio flux and a factor of $\sim 4$ deeper in X-ray flux. Moreover, while very few previously known FSRQ had relatively large X-ray-to-radio flux ratios $\left(f_{\mathrm{x}} / f_{\mathrm{r}}>10^{-11.5}\right.$ in the units of Fig. 1: see the dashed line), many DXRBS FSRQ are quite "X-ray bright."

This is better seen in Fig. 2, which plots radio versus X-ray luminosity for FSRQ. About $25 \%$ of DXRBS FSRQ have $L_{\mathrm{x}} / L_{\mathrm{r}} \gtrsim 10^{-6}$ (or, alternatively, $\left.\alpha_{\text {rx }} \lesssim 0.78\right)$. Only nine $1 \mathrm{Jy} / \mathrm{S} 4$ FSRQ had such luminosity ratios. Based on the overall spectral energy distribution of these sources and extrapolating from the situation in BL Lacs, these "X-ray bright" FSRQ probably have the peak of their synchrotron emission at UV/X-ray energies, unlike the other, more common, FSRQ, which peak in the IR/optical band. Due to their lower radio fluxes, DXRBS FSRQ are also reaching relatively low radio luminosities, approaching

\footnotetext{
${ }^{2}$ Note that it can be difficult to distinguish between a BL Lac and a radio galaxy for border-line sources. Therefore, while we are confident that the classification criteria have been applied consistently for our newly discovered blazars, this might not be the case for the previously known sources.
} 


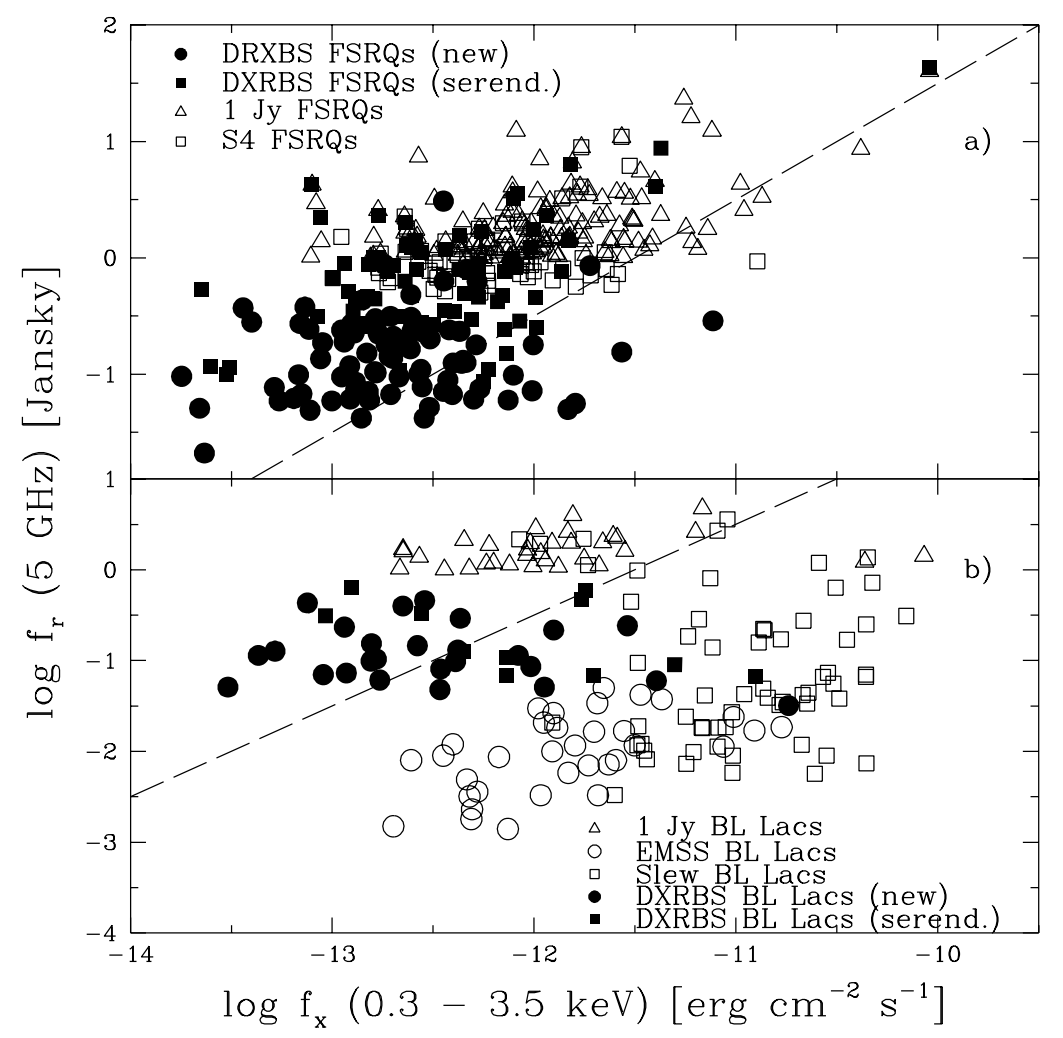

Fig. 1. The radio/X-ray flux plane for FSRQ (a); top) and BL Lac (b); bottom) samples. Note how DXRBS is sampling previously unexplored regions of parameter space. The dashed lines correspond to $\log f_{\mathrm{x}} / f_{\mathrm{r}}=-11.5$.

what should be the lower end of the FSRQ luminosity function according to unified schemes $\left(L_{\mathrm{r}} \approx 10^{24.5} \mathrm{~W} \mathrm{~Hz}^{-1}\right.$ : Urry \& Padovani 1995). In particular, more than $20 \%$ of them have $L_{\mathrm{r}}<10^{26.5} \mathrm{~W} \mathrm{~Hz}^{-1}$, as compared to only $3 \%$ for the $1 \mathrm{Jy}$ and $\mathrm{S} 4$ samples.

As regards BL Lacs, DXRBS is again exploring uncharted territory, as it is finding BL Lacs which cover a previously unexplored region of the radio/Xray plane, and go deeper (by almost an order of magnitude) in X-ray flux than currently available samples (see Fig. 1b). Note also how the DXRBS BL Lacs are intermediate in their X-ray-to-radio flux ratios as compared to the "classical" radio and X-ray selected samples. A more complete description of the DXRBS first results is given by Perlman et al. (1998). 


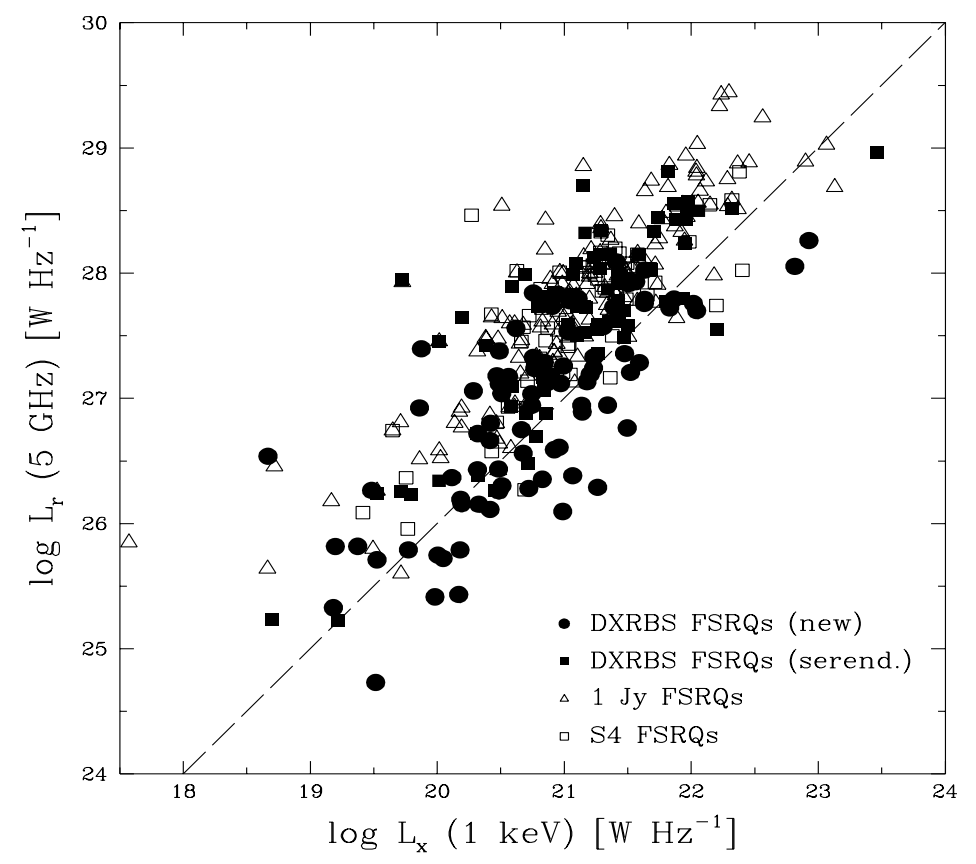

Fig. 2. The radio and X-ray luminosities of FSRQ.

\section{References}

Blandford, R., D., \& Rees, M. J. (1978): Pittsburgh Conference on BL Lac Objects, A. N. Wolfe ed., 328

Condon, J. J., et al., (1997): preprint (http://www.nrao.edu)

Drinkwater, M. J., Barnes, D. G., \& Ellison, S. L. (1995): PASA 13, 12

Drinkwater, M. J., et al. (1997): MNRAS 284, 85

Gregory, P. C., Scott, W. K., Douglas, K., \& Condon, J. J. (1996): ApJS 103, 427.

Griffith, M. R., \& Wright, A. E. (1993): AJ 106, 1095

Irwin, M., Maddox, S. \& McMahon, R. (1994): Spectrum 2, 14

Laurent-Muehleisen, S. A., Kollgaard, R. I., Ciardullo, R., Feigelson, E. D., Brinkmann, W., \& Siebert, J. (1997): preprint (astro-ph/9711268)

Maccacaro, T., et al. (1994): Astrop. Lett. \& Communications 29, 267

Padovani, P. (1997): Very High Energy Phenomena in the Universe, Y. Giraud-Héraud, J. Trân Thanh Vân eds., Editions Frontieres, 7

Perlman, E. S., et al. (1996): ApJS 104, 251

Perlman, E. S., Padovani, P., Giommi, P., Sambruna, R., Jones, L. R., Tzioumis, A., \& Reynolds, J. (1998): AJ 116, in press

Stickel, M., Padovani, P., Urry, C. M., Fried, J. W., \& Kühr, H. (1991): ApJ 374, 431.

Urry, C. M., \& Padovani, P. (1995): PASP 107, 803 
Wall, J. V., \& Peacock, J. A. (1985): MNRAS 216, 173

White, R. L., \& Becker, R. H. (1992): ApJS 79, 331

White, N. E., Giommi, P., \& Angelini, L. (1995):

http://heawww.gsfc.nasa.gov/users/white/wgacat/wgacat.html

Wolter, A., Caccianiga, A., Della Ceca, R., Gioia, I. M., Maccacaro, T., \& Ruscica, C. (1997): From the Micro- to the Mega-Parsec, A. Comastri, T. Venturi, M. Bellazzini eds., Mem. Soc. Astron. Ital. 68, 147 